\title{
Towards Quantitative Nanomagnetism in Transmission Electron Microscope by the Use of Patterned Apertures
}

Hasan $\mathrm{Ali}^{1,3, *}$, Tobias Warnatz ${ }^{2}$, Ling Xie ${ }^{1}$, Björgvin Hjörvarsson ${ }^{2}$ and Klaus Leifer ${ }^{1}$

1. Applied Materials Science, Department of Engineering Sciences, Uppsala University, Box 534, 75121

Uppsala, Sweden

2. Department of Physics and Astronomy, Uppsala University, Box 516, 75120 Uppsala, Sweden

${ }^{3 .}$ Department of Electrical Engineering, Mirpur University of Science and Technology, 10250 Mirpur (AJK), Pakistan

* Corresponding author: Hasan.Ali@angstrom.uu.se

Electron magnetic circular dichroism (EMCD) is an electron energy loss spectroscopy (EELS) based technique to measure the magnetic properties of the materials in transmission electron microscope. The technique was introduced in 2003[1] and over the last decade it has seen a continuous rise with spatial resolution reaching atomic planes[2]. Although EMCD has the potential to get magnetic information down to atomic level but there are still some crucial challenges which make the quantitative EMCD analysis very difficult. EMCD has a notoriously bad signal to noise $(\mathrm{S} / \mathrm{N})$ ratio. In EMCD experiments, the TEM sample is quite highly oriented to fulfill 2 beam (or 3 beam) condition and two EELS spectra are acquired at two different scatter angles. In most of the cases, the EELS spectra or the maps of EELS spectra are acquired one by one by moving the aperture from one position to the other and hence it cannot be guaranteed that the experimental conditions are the same for both acquisitions. For example, properties of the specimen may change significantly due to beam damage [3] and the sample drift can limit the spatial resolution of the analysis [4]. Moreover unlike the x-ray based technique XMCD, EMCD is highly dependent on sample orientation. It is known by simulations that a small change in orientation of the sample may significantly alter the EMCD signal [5] but no experimental study has been done in this context to establish a quantitative relationship between the change in sample orientation and its effect on the resulting EMCD signal.

We designed special apertures which can help to eliminate the problems associated with the quantitative EMCD analysis. First we built a double hole aperture (DA) to simultaneously acquire the two EELS spectra required for EMCD which removes all the complications produced due to the serial acquisition of the spectra. We show that we get a much better $\mathrm{S} / \mathrm{N}$ ratio and the signal purity using the DA as compared to the previously used qE-mode for this purpose [6]. Second we built a special quadruple aperture (QA) to simultaneously acquire the EELS spectra and the sample orientation.

To experimentally demonstrate the technique, we used a $25 \mathrm{~nm}$ thick film of bcc Fe grown on $\mathrm{MgO}$ (001) substrate. Fig. 1a shows the electron diffraction pattern (DP) with the sample tilted in a 2 beam condition (2BC). The beams are aligned parallel to the $\mathrm{q}_{\mathrm{x}}$-axis in the reciprocal space whereas the aperture holes are aligned parallel to the $\mathrm{q}_{\mathrm{y}}$-axis (Fig. 1b). A CCD acquisition in the spectroscopy mode then produces the image of two EELS spectra (Fig. 1c) where the $q_{x}$ is replaced by the energy dispersion axis whereas the values along $\mathrm{q}_{\mathrm{y}}$ are preserved. By integrating the intensity along the boxes shown in Fig. 1c , the EELS spectra are extracted and the EMCD signal is obtained by taking the difference of the two EELS spectra. The acquisition time for Fig. 1c was 60s. It can be seen that the resulting EMCD 
signal is almost noise free with a well-defined signal peaks at L3 and L2 edges. Applying the sum rules, we calculate a $\mathrm{m}_{\mathrm{l}} / \mathrm{m}_{\mathrm{s}}$ ratio of $0.06 \pm 0.01$.

To demonstrate the QA experiment, a $2 \mathrm{~nm}$ wide electron probe was scanned across the Fe film and a CCD image was acquired at each scan point in the spectroscopy mode. Fig. 2 shows the date acquired at three different scan points. Figure 2c shows the QA-image acquired at one of the scan points. The middle two spectra are used to extract the EMCD signal whereas the upper and the lower spectra are used to determine the intensity of the direct beam $\left(\mathrm{I}_{0}\right)$ and the diffracted beam $\left(\mathrm{I}_{\mathrm{g}}\right)$. A change in orientation can be estimated by a change in $\mathrm{I}_{0} / \mathrm{I}_{\mathrm{g}}$ ratio. Figure 2 (d,e,f) shows the EELS spectra extracted for three different scan points along with the corresponding $I_{0} / I_{g}$ ratio. It can be seen that the sample orientation is changing quite significantly between different points. Qualitatively, the less the difference between the intensities of $\mathbf{0}$ and $\mathbf{g}$ beams, the better the EMCD signal and vice versa. More experiments are needed to develop a quantitative relationship between the change in orientation and its influence on the resulting signal which will be done in near future.

[1] P. Schattschneider et al., "Detection of magnetic circular dichroism using a transmission electron microscope,” Nature, vol. 441, no. 7092, pp. 486-488, May 2006.

[2] J. Rusz et al., "Magnetic measurements with atomic-plane resolution,” Nat. Commun., vol. 7, no. May, p. 12672, 2016.

[3] R. F. Egerton et al., "Radiation damage in the TEM and SEM."

[4] T. Thersleff et al., "Detection of magnetic circular dichroism with subnanometer convergent electron beams,” Phys. Rev. B, vol. 94, no. 13, p. 134430, Oct. 2016.

[5] H. Lidbaum et al., "Reciprocal and real space maps for EMCD experiments," Ultramicroscopy, vol. 110, pp. 1380-1389, 2010.

[6] H. Ali et al., "Quantitative EMCD by use of a double aperture for simultaneous acquisition of EELS,” Ultramicroscopy, vol. 196, pp. 192-196, Jan. 2019.
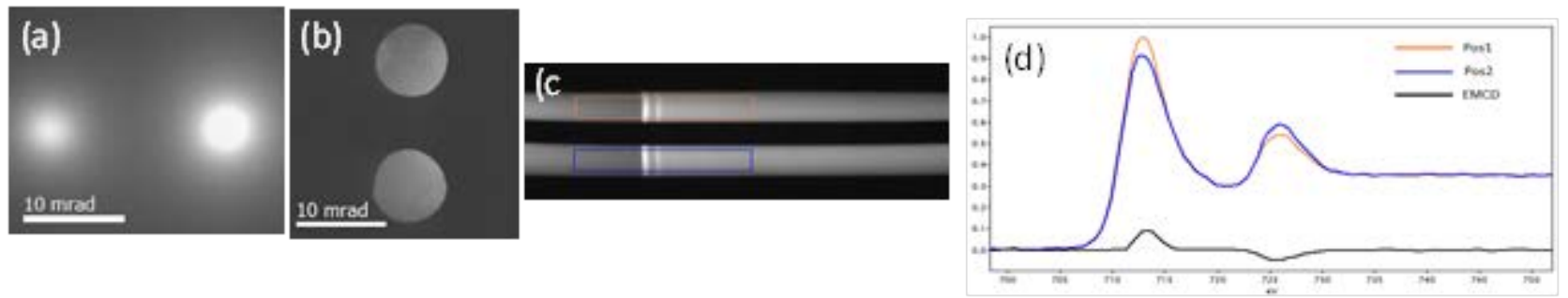

Figure 1: (a) Electron DP with the sample tilted in 2BC (b) the image of the DA (c) image of the two EELS spectra (d) EELS spectra extracted from (c) and their difference (EMCD) signal
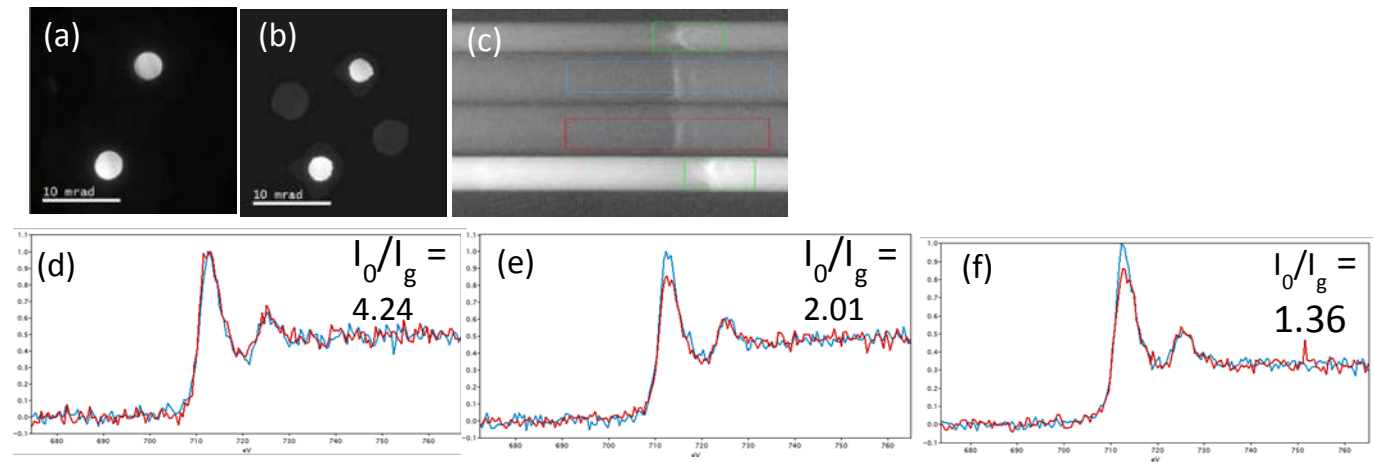

Figure 2: (a) Electron DP in 2BC (b) QA inserted in the diffraction plane (c) 2D image acquired at each scan point (d,e,f) EELS spectra extracted from the middle two spectra in (c) for three different points. 